\title{
Microscopic screening of cyclodextrin channel blockers by DiffusiOptoPhysiology
}

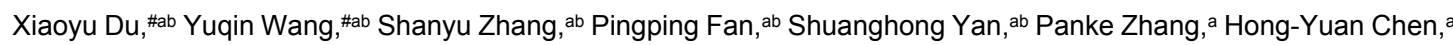
and Shuo Huang ${ }^{*}$ ab

\# These authors contributed equally to this work

a State Key Laboratory of Analytical Chemistry for Life Sciences, School of Chemistry and Chemical Engineering, Nanjing University, 210023, Nanjing, China.

${ }^{\mathrm{b}}$ Chemistry and Biomedicine Innovation Center (ChemBIC), Nanjing University, 210023, Nanjing, China.

${ }^{*}$ Corresponding author. Email: shuo.huang@nju.edu.cn.

\section{Table of Content}

$\begin{array}{llr}\text { Figure S1. The 4-well DOP chip. } & \text { S-2 }\end{array}$

Figure S2. A representative fluorescence time trace acquired by DOP using WT $\alpha-H L$. S-3

$\begin{array}{lll}\text { Figure S3. Definition of parameters. } & S-4\end{array}$

Figure S4. Event statistics of DM- $\beta-C D$ and TM- $\beta-C D$ sensing.

Figure S5. DM- $\beta-C D$ and TM- $\beta-C D$ blockages to multiple WT $\alpha-H L$ nanopores.

$\begin{array}{llr}\text { Figure S6. The multiplex DOP chip. } & \text { S-7 }\end{array}$

$\begin{array}{ll}\text { Figure S7. Purification and characterization of } \alpha-H L ~ M 113 F . & \text { S-8 }\end{array}$

$\begin{array}{ll}\text { Figure S8. The bright-field image of a DIB array. } & \text { S-9 }\end{array}$

Figure S9. Event statistics of DM- $\beta-C D$ blockage to a WT $\alpha-H L$ during electrophysiology recordings. $\quad$ S-10

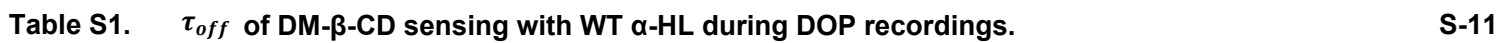

$\begin{array}{lll}\text { Table S2. } & \tau_{\text {off }} \text { of TM- } \beta-C D \text { sensing with WT } \alpha-H L \text { during DOP recordings. } & \text { S-11 }\end{array}$ 


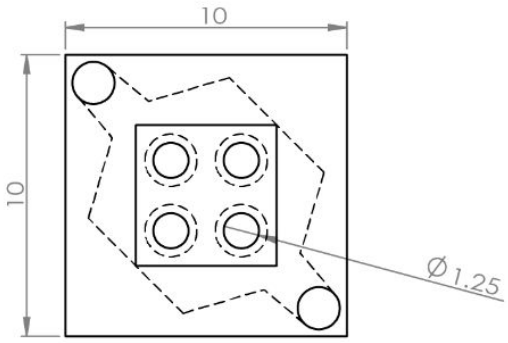

b

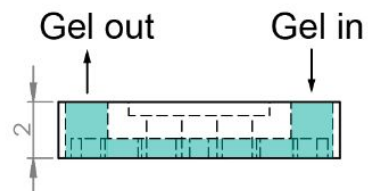

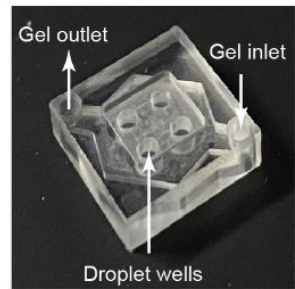

C

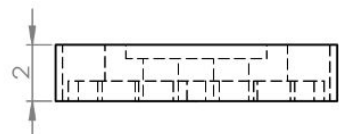

Figure S1. The 4-well DiffusiOptoPhysiology (DOP) chip. The chip was custom manufactured with polymethyl methacrylate (PMMA). (a) The top view of the chip. (b) The front view of the chip. The molten gel is filled into the device along the route marked in green. (c) The side view of the chip. Scale unit in a-c: $\mathrm{mm}$. (d) The actual DOP chip. The gel inlet and outlet holes are marked on the photo. The gel outlet also helps air bubbles to escape from the device during gel filling. The demonstrated DOP device contains four droplet wells, capable of performing four parallel measurements with the same chip. 

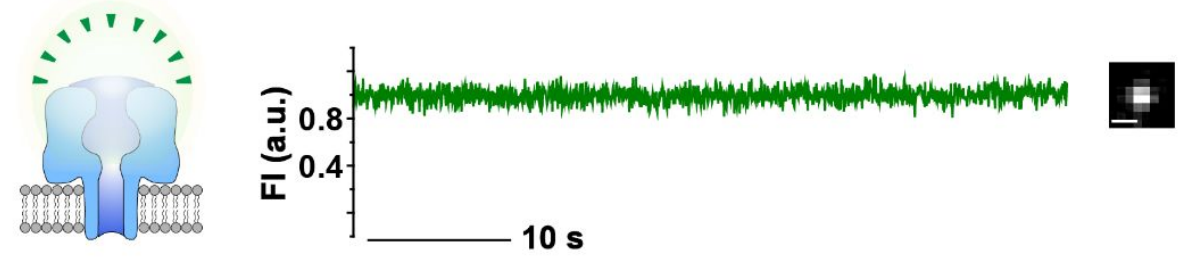

Figure S2. A representative fluorescence time trace acquired by DOP using wild-type $\alpha$-hemolysin (WT $\alpha-H L)$. Left: the schematic diagram of a single WT $\alpha-H L$ inserted in a droplet interface bilayer (DIB). Right: a representative fluorescence time trace. A representative frame containing a nanopore at its open state is placed to the right of the trace. Scale bar: $4 \mu \mathrm{m}$. The DOP recording was performed with $1.5 \mathrm{M} \mathrm{KCl}, 400 \mu \mathrm{M}$ EDTA, $33 \mu \mathrm{M}$ Fluo-8, $10 \mathrm{mM}$ HEPES ( $\mathrm{pH} 7.0)$ in cis and $0.75 \mathrm{M} \mathrm{CaCl}_{2}, 10 \mathrm{mM}$ HEPES ( $\left.\mathrm{pH} 7.0\right)$ in trans. 


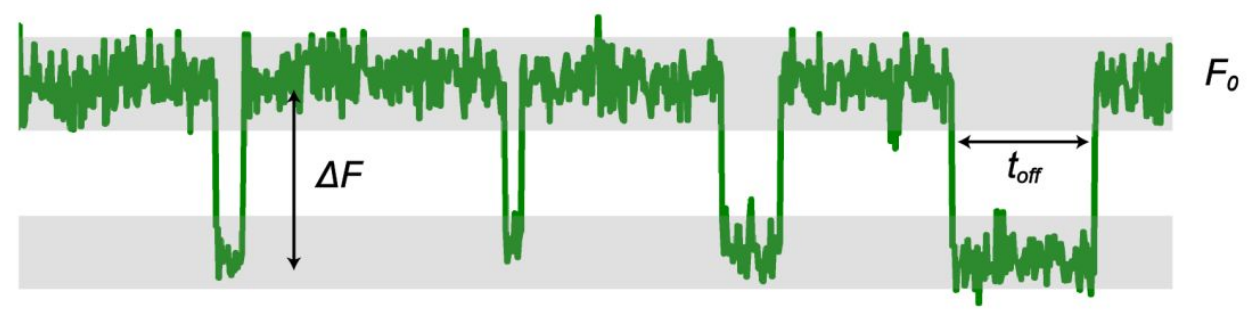

$5 \mathrm{~s}$

Figure S3. Definition of parameters. A representative fluorescence trace acquired with DOP. The event dwell time $\left(t_{o f f}\right)$, the open pore fluorescence intensity $\left(F_{0}\right)$ and the blockade fluorescence intensity $(\Delta F)$ were defined as marked on the trace. For the demonstration, DOP was carried out with a WT $\alpha-H L$. Trimethyl- $\beta$-cyclodextrin (TM- $\beta-C D)$ was applied as the channel blocker. 

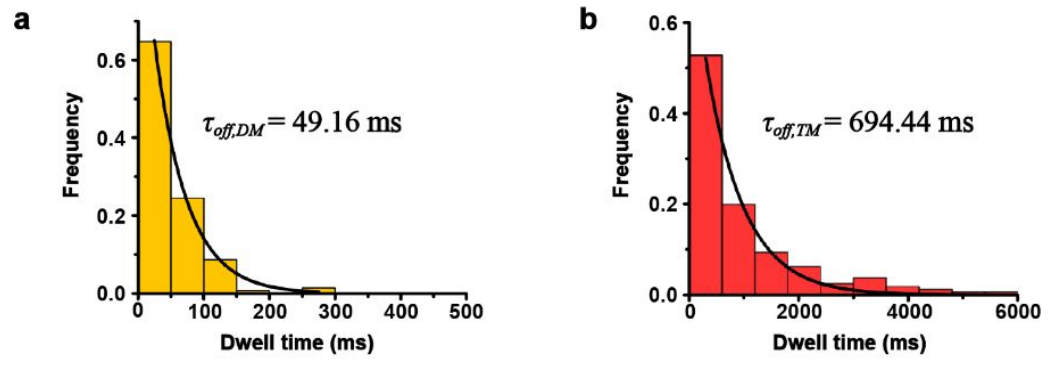

Figure S4. Event statistics of 2,6-di-O-methyl- $\beta$-cyclodextrin (DM- $\beta-C D)$ and TM- $\beta-C D$ sensing. Both sets of results were acquired with WT $\alpha-\mathrm{HL}$ by DOP. The measurements were performed with $1.5 \mathrm{M} \mathrm{KCl}, 400 \mu \mathrm{M}$ EDTA, 33 $\mu \mathrm{M}$ Fluo-8, $10 \mathrm{mM}$ HEPES ( $\mathrm{pH} 7.0)$ in cis and $0.75 \mathrm{M} \mathrm{CaCl}_{2}, 10 \mathrm{mM} \mathrm{HEPES}(\mathrm{pH} 7.0)$ in trans. (a-b) The histogram of the event dwell time when $10 \mathrm{mM}$ DM- $\beta-C D$ (a) or $89.3 \mathrm{mM}$ TM- $\beta-C D$ (b) was respectively applied as the channel blocker. The mean dwell time $\tau_{o f f, D M}$ (a) or $\tau_{o f f, T M}$ (b) was respectively derived from results of the single exponential fitting following the equation $y=a * \exp (-x / \tau)$. 

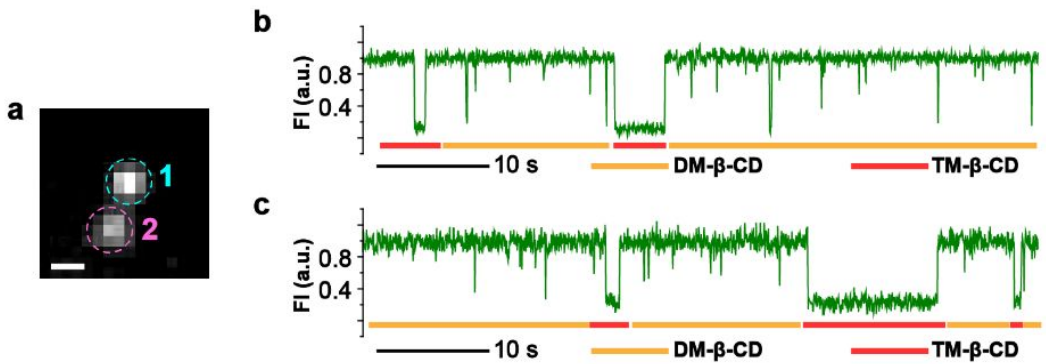

Figure S5. DM- $\beta-C D$ and TM- $\beta-C D$ blockages to multiple WT $\alpha-H L$ nanopores. (a) A representative frame acquired during DOP. Two $\alpha-\mathrm{HL}$ pores respectively named 1 (marked by cyan circle) and 2 (marked by pink circle) were simultaneously monitored in the same field of view. Scale bar: $4 \mu \mathrm{m}$. (b-c) The fluorescence time traces acquired from pore 1 (b) or pore 2 (c). DM- $\beta-C D$ and TM- $\beta-C D$ were simultaneously sensed as a mixture. The yellow lines mark the events of $D M-\beta-C D$ while the red lines mark the events of TM- $\beta-C D$ 
a
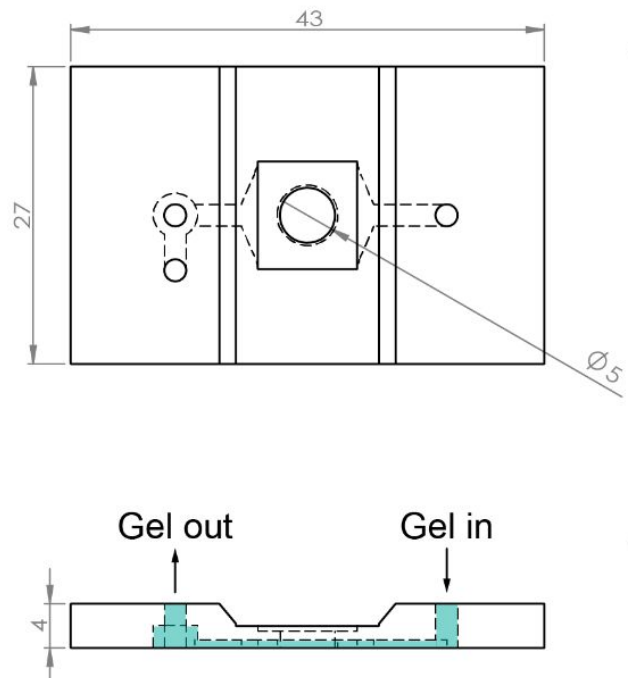

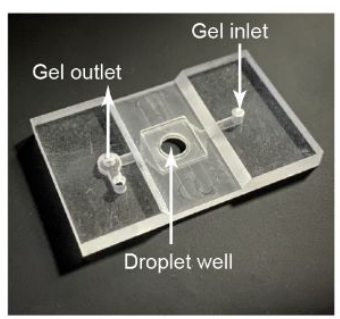

C

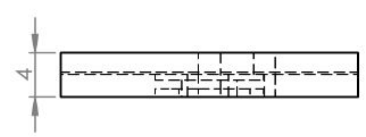

Figure S6. The multiplex DOP chip. The chip was custom manufactured with polymethyl methacrylate (PMMA). (a) The top view of the chip. (b) The front view of the chip. The molten gel is filled into the device along the route marked in green. (c) The side view of the chip. Scale unit in a-c: $\mathrm{mm}$. (d) The actual DOP chip. The gel inlet and outlet holes are marked on the photo. The extra hole on the outlet side helps air bubbles to escape from the chip during gel filling. The demonstrated DOP device contains one big droplet well, capable of holding multiple droplets for multiplex measurements. 


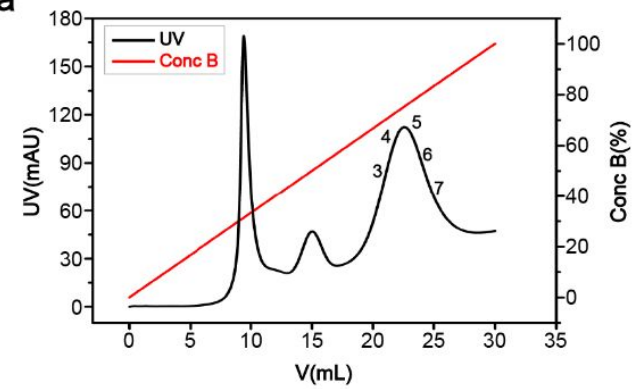

b

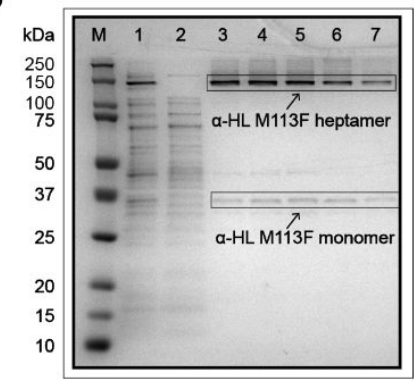

Figure S7. Purification and characterization of $\alpha-H L$ M113F. (a) The UV absorbance spectrum during column elution of $\mathrm{Ni}$-affinity chromatography purification. Briefly, the supernatant of the bacterial lysate was loaded on a nickel affinity column. During buffer elution with a gradient of imidazole (0-500 mM), non-specific protein, the $\alpha-H L$ M113F monomer and the heptamer were sequentially eluted based on their difference in the binding affinities to the nickel affinity column (HisTrapTM HP, GE Healthcare). The identities of the marked samples were confirmed by gel electrophoresis. (b) Gel electrophoresis of different elution fractions during the purification of $\alpha-\mathrm{HL}$ M113F on a 12\% SDS-PAGE gel. Lanes: M, precision plus protein standards (Bio-Rad); 1 , the supernatant of the bacteria lysate; 2 , the eluted sample from the nickel affinity column immediately after bacteria lysate loading; $\alpha-H L M 113 F$ in the lysate would bind to the column and the corresponding bands have disappeared. 3-7, the corresponding elution fractions in (a). According to previously published results ${ }^{1}$, the $\alpha-H L$ M113F heptamer was confirmed in fraction 3-7. 


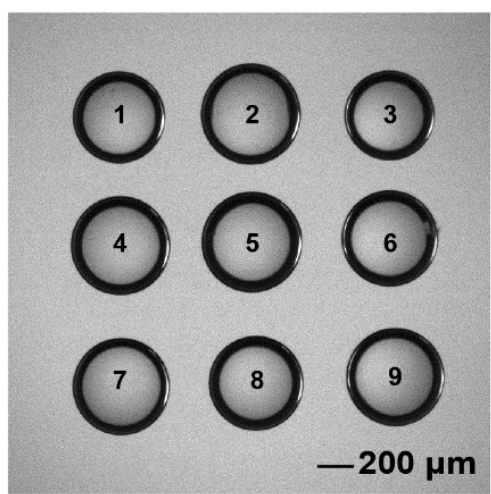

Figure S8. The bright-field image of a DIB array. To accommodate more DIBs in the same chip, a multiplex DOP chip (Figure S6) was applied. In this bright-field image, 9 DIBs were showed. Different droplets, as marked with different indices, contain different types of pores and channel blockers. 1 represents a DIB formed by a droplet containing no pore and blocker. 2 and 3 respectively represent WT $\alpha-H L$ and $\alpha-H L$ M113F without any blockers in the droplet. 4, 5 and 6 respectively contain $\beta$-cyclodextrin $(\beta-C D), D M-\beta-C D$ and $T M-\beta-C D$ as the channel blockers. Whereas, they all contain WT $\alpha-H L .7,8$ and 9 respectively contain $\beta-C D, D M-\beta-C D$ and TM- $\beta-C D$ as the channel blockers. Whereas, they all contain $\alpha-H L$ M113F. 


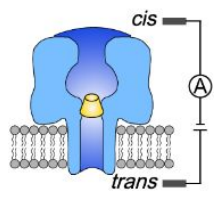

C

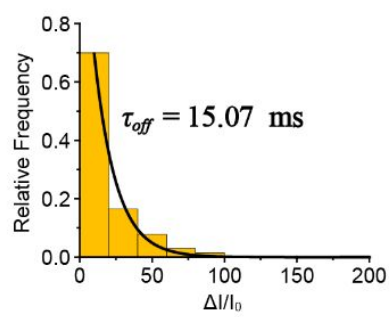

b

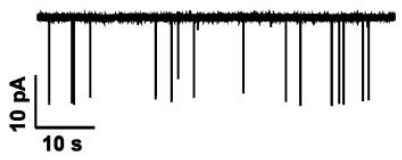

d

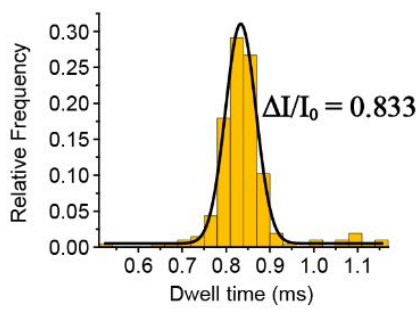

Figure S9. Event statistics of DM- $\beta-C D$ blockage to a WT $\alpha-H L$ during electrophysiology recordings. (a) The schematic diagram of DM- $\beta-C D$ blockage to a WT $\alpha-H L$ (yellow cone). (b) Representative electrophysiological trace corresponding to DM- $\beta-C D$ blockage to a WT $\alpha-\mathrm{HL}$ (c) The histogram of the event dwell time of DM- $\beta-C D$ blockage to a WT $\alpha-H L$. (d) The histogram of the relative blockade depth $\left(\Delta I / I_{0}\right)$ of DM- $\beta-C D$ blockage to a WT $\alpha-H L$. The peak value $\Delta \boldsymbol{I} / \boldsymbol{I}_{\mathbf{0}}$ was derived from the Gaussian fitting results. The equation to perform Gaussian fitting is $\boldsymbol{y}=\boldsymbol{y}_{\mathbf{0}}+\boldsymbol{A} * \boldsymbol{\operatorname { e x p }}$ $\left(-\left(\boldsymbol{x}-\boldsymbol{x}_{\boldsymbol{c}}\right)^{2} /\left(2 * \boldsymbol{w}^{2}\right)\right), \Delta I / I_{0}=x_{c}$. The time constant $\tau_{\text {off }}$ was derived from the corresponding single exponential fitting results, following the equation $y=a * \exp (-x / \tau)$. DM- $\beta-C D$ was added to cis with a $6 \mathrm{mM}$ final concentration. The electrophysiology recording was performed with $1.5 \mathrm{M} \mathrm{KCl}, 400 \mu \mathrm{M}$ EDTA, $10 \mathrm{mM} \mathrm{HEPES}(\mathrm{pH} 7.0)$ in cis and 0.75 $\mathrm{M} \mathrm{CaCl}_{2}, 10 \mathrm{mM}$ HEPES (pH 7.0) in trans. The demonstrated trace was recorded with a $+20 \mathrm{mV}$ continuously applied voltage. 
Table S1: $\tau_{\text {off }}$ of DM- $\beta-C D$ sensing with WT $\alpha-H L$ during DOP recordings. The DIB was established with $1.5 \mathrm{M}$ $\mathrm{KCl}, 400 \mu \mathrm{M}$ EDTA, $33 \mu \mathrm{M}$ Fluo-8, $10 \mathrm{mM}$ HEPES, $\mathrm{pH} 7.0$ in cis and $0.75 \mathrm{M} \mathrm{CaCl}_{2}, 10 \mathrm{mM}$ HEPES, pH 7.0 in trans. DM- $\beta-C D$ was added to cis with a final concentration of $10 \mathrm{mM}$. Three independent measurements were performed and the corresponding results are listed below.

\begin{tabular}{ccc}
\hline Individual experiments & $\boldsymbol{\tau}_{\text {off }}(\mathbf{m s})$ \\
\hline $\mathbf{1}$ & 43.50 \\
$\mathbf{2}$ & 49.16 \\
$\mathbf{3}$ & 31.89 \\
\hline
\end{tabular}

Table S2: $\boldsymbol{\tau}_{\text {off }}$ of TM- $\beta-C D$ sensing with WT $\boldsymbol{\alpha}-\mathrm{HL}$ during DOP recordings. The DIB was established with $1.5 \mathrm{M}$ $\mathrm{KCl}, 400 \mu \mathrm{M}$ EDTA, $33 \mu \mathrm{M}$ Fluo-8, $10 \mathrm{mM}$ HEPES, pH 7.0 in cis and $0.75 \mathrm{M} \mathrm{CaCl}_{2}, 10 \mathrm{mM}$ HEPES, pH 7.0 in trans. TM- $\beta-C D$ was added to cis with a final concentration of $89.3 \mathrm{mM}$. Three independent measurements were performed and the corresponding results are listed below.

\begin{tabular}{cc}
\hline Individual experiments & $\boldsymbol{\tau}_{\text {off }}(\mathbf{m s})$ \\
\hline $\mathbf{1}$ & 653.59 \\
$\mathbf{2}$ & 694.44 \\
$\mathbf{3}$ & 636.94 \\
\hline
\end{tabular}




\section{References}

1. Wang, Y.; Yan, S.; Zhang, P.; Zeng, Z.; Zhao, D.; Wang, J.; Chen, H.; Huang, S. Osmosisdriven motion-type modulation of biological nanopores for parallel optical nucleic acid sensing. ACS Appl. Mater. Interfaces 2018, 10, 7788-7797. 